\section{A DRAM-atic end for T cells}

Infection of $\mathrm{CD}^{+}{ }^{+} \mathrm{T}$ cells with human immunodeficiency virus (HIV) can result in a caspase-independent cell-death pathway, but the details of this process remain unclear. In PLoS Pathogens, Estaquier and colleagues link the autophagy-regulatory protein DRAM to HIV-triggered death of CD4 ${ }^{+} \mathrm{T}$ cells. Infection of T cells with HIV induces DRAM expression in a manner dependent on the tumor suppressor p53 and subsequent activation of autophagy and cell death. However, autophagy is not critical for cell death; instead, after infection with HIV, DRAM associates with lysosomes and initiates destabilization of their membranes-a classic cellintrinsic death initiator. How DRAM actually disrupts lysosomal membranes is unclear, but the cell death that results from DRAM activity substantially impairs viral infection. DRAM-mediated killing may therefore represent a cell-intrinsic mechanism for eliminating virus-infected cells.

ZF

PLoS Pathog. (2 May 2013) doi:10.1371/journal.ppat.1003328

\section{Becoming pandemic}

Mutant-selection experiments with the highly pathogenic avian influenza virus $\mathrm{H} 5 \mathrm{~N} 1$ have identified a virus that is transmissible to ferrets. In Nature, Xiong et al. report that the transmissible mutant virus has slightly higher binding affinity of hemagglutinin (HA) for its human receptor (sialic acid in $\alpha 2,6$-linkage to galactose on sugar side chains) and much less affinity for the avian receptor (sialic acid in $\alpha 2,3$-linkage). The crystal structure of the transmissible mutant HA in complex with receptor analogs shows that the mutant HA binds human receptors in the same folded-back conformation as did HA from viruses in the pandemics of 1918 (H1), 1957 (H2) and 1968 (H3), although the binding is weaker. This binding mode is different from that of wild-type H5 HA and arises from conformational changes due to a Q226L point substitution. The structural consequences of this substitution are similar to those of the pandemic viruses $\mathrm{H} 2$ and $\mathrm{H} 3$.

Nature 497, 392-396 (2013)

\section{Mast cell-DC axis}

Malaria continues to pose a considerable global health threat; hence, understanding the immune response to plasmodia is of utmost importance. In Nature Medicine, Guermonprez et al. describe a mast cell-dendritic cell (DC) axis associated with plasmodia infection. Infection of mice with Plasmodium chabaudi leads to release of the cytokine FIt3L from mast cells, which substantially increases the number of CD8 $\alpha^{+}$DCs and, in turn, activates $C D 8^{+} \mathrm{T}$ cells. The authors trace those effects to higher expression of xanthine dehydrogenase in a yetto-be identified radiosensitive cell population. That population responds to type I interferons produced during infection.

Plasmodia-infected erythrocytes release hypoxanthine, which then can be converted to uric acid by xanthine dehydrogenase. Uric acid triggers mast cell degranulation and cleavage of membrane-tethered FIt3L. A similar response occurs in human patients with malaria, which suggests that this innate response to plasmodia is conserved. $L A D$ Nat. Med. (19 May 2013) doi:10.1038/nm.3197

\section{Systemic alarmin} membrane is important. B cells use actomyosin contractility to pull out and invaginate the presenting membranes and internalize the antigen through clathrin- and actin-dependent processes. Diminishing the strength of myosin Ila contraction improves the internalization of low-affinity antigens. This suggests that although bonds between the B cell receptor (BCR) and antigen in small microclusters might break under the pulling forces, larger microclusters resist the contractile forces for long enough (20-30 seconds) to allow the association of clathrin and internalization. Thus, contractile forces mechanically test the strength of BCR binding and provide a mechanism for affinity discrimination. Science (16 May 2013) doi:10.1126/science.1237572
Cochlin, a glycosylated extracellular matrix protein found in the inner ear, is also expressed in the spleen, but its physiological role there remains unknown. In Immunity, Py et al. show cochlin is secreted by follicular DCs. After bacterial infection, cochlin is proteolytically cleaved by aggrecanase 1 (whose expression is induced by tumor-necrosis factor), which results in the release of a bioactive amino-terminal fragment that functions systemically to activate innate immune responses. $\mathrm{Coch}^{-/-}$ mice have normal splenic and lymph node structure and adaptive immunity but fail to control infection with Pseudomonas or Staphylococcus. A defect in the recruitment of monocytes and neutrophils leads to greater bacterial burdens, which ultimately kills the $\mathrm{Coch}^{-/-}$mice. Thus, cleavage of cochlin releases a systemic alarmin that contributes to innate immunity.

Immunity 38, 1063-1072 (2013)
$L A D$ 